\title{
Uncovering Chromatin's Contribution to the Mitotic Spindle: Applications of Computational and Polymer Models
}

\author{
Matthew E. Larson, Benjamin D. Harrison, and Kerry Bloom \\ Department of Biology, University of North Carolina-Chapel Hill, CB\# 3280, Coker Hall, Chapel \\ Hill, North Carolina 27599-3280, USA
}

\begin{abstract}
The mitotic spindle is a structure that forms during mitosis to help ensure that each daughter cell receives a full complement of genetic material. In metaphase, the spindle contains microtubules that nucleate inward from two opposing poles. Chromosomes are attached to plus-ends of these microtubules via protein structures called kinetochores. The centromere is the specific region of kinetochore attachment on the chromosome. Chromatin surrounding the centromere (pericentric chromatin) is subject to microtubule-based forces and is commonly modeled as a linear spring, where the force that it exerts is proportional to the distance that it is stretched. We have incorporated physically based models of chromatin to create more accurate and predictive models of the spindle. In addition, using fluorescence microscopy and motion analysis of fluorescently labeled chromatin spots we discovered that pericentric chromatin is restrained relative to free diffusive motion. The characterization of chromatin is crucial to understand mitotic spindle stability and to understand the cell cycle checkpoint regulating anaphase onset.
\end{abstract}

\section{Keywords}

pericentric chromatin; worm-like chain; chromatin dynamics; modeling of chromatin

\section{Introduction}

The mitotic spindle is an apparatus that forms during the division of eukaryotic cells. Proper function of the mitotic spindle is essential to the accurate segregation of chromosomes, which ensures that daughter cells have all of the genetic information necessary for survival [1]. The structure and organization of the mitotic spindle is highly conserved throughout eukaryotic organisms. Thus, a complete understanding of the structure and function of the mitotic spindle is essential for a complete picture of life and, in the future, may yield many novel drug targets for cancer and other diseases where improper or unregulated cell division is implicated.

At metaphase, the mitotic spindle is a highly symmetric structure, consisting of two microtubule organizing centers (known as spindle poles in the budding yeast Saccharomyces cerevisiae, or centrosomes in vertebrate cells), from which two categories of microtubules

\footnotetext{
(C) 2010 Elsevier Masson SAS. All rights reserved.

*Corresponding Author: kbloom@email.unc.edu, Tel: +1 919962 1182, Fax: +1 9199621625.
}

Publisher's Disclaimer: This is a PDF file of an unedited manuscript that has been accepted for publication. As a service to our customers we are providing this early version of the manuscript. The manuscript will undergo copyediting, typesetting, and review of the resulting proof before it is published in its final citable form. Please note that during the production process errors may be discovered which could affect the content, and all legal disclaimers that apply to the journal pertain. 
grow inward. The first of these are the interpolar microtubules, which overlap in the center of the mitotic spindle. These microtubules are held together in an antiparallel fashion through microtubule-based motor proteins, known as kinesins. Microtubule plus-end directed kinesins (Cin8 and Kip1) [2] generate a net outward force on antiparallel microtubule arrays, driving spindle elongation. The second type of microtubules emanating from the spindle poles are kinetochore microtubules that provide physical connection to the replicated sister chromatids through proteinaceous structures known as kinetochores [3]. During metaphase, the sister chromatids are aligned at the center of the mitotic spindle, a region commonly known as the metaphase plate (Fig. 1). In the budding yeast

Saccharomyces cerevisiae, one microtubule attaches to each sister chromatid [4] through the kinetochore at a designated DNA sequence known as the centromere [5]. In more complex eukaryotes, such as humans, multiple microtubules associate with a single sister chromatid. The streamlined structure of the yeast mitotic spindle, combined with the ease of genetic manipulations in yeast, make it an ideal organism within which to study the structure and biophysical properties of the mitotic spindle components.

During metaphase this mitotic spindle attains a steady-state length, suggesting that the outward microtubule-based motor force is balanced by an inward force provided by the bioriented sister chromatids [6]. Elastic recoil of chromatin DNA has been observed in vivo [7], suggesting that microtubules are indeed capable of exerting tensile force on the sister chromatids and that the release of this force can cause the chromatids to recoil poleward. These experiments also demonstrate the highly viscous nature of the environment in the cell. This environment is so viscous that inertia is inconsequential (i.e. low Reynolds number situation).

In many force-balanced based mathematical models of the mitotic spindle, the paired sister chromatids are assumed to exert a force that is linearly proportional to extension length [8]. However, in vitro experiments have demonstrated that reconstituted chromatin responds in a complex manner when tensile stresses are applied [9]. It is impossible to faithfully reconstruct chromatin and the intracellular environment in vitro. Thus, even though in vitro experiments have been useful in demonstrating that chromatin responds in a complex manner to applied tension, in vivo measurements are needed to accurately determine the tensile force that the chromatin can produce. Here, we attempt to explain the behavior of the chromatin as a complex polymer in order to more accurately model its behavior and increase the predictive power of mathematical and computational models of the mitotic spindle.

In depth analyses of biopolymers have been used to determine some of their mechanical properties [10]. These analyses, coupled with super-resolution fluorescence microscopy, provide the tools needed to examine the mechanical properties of the mitotic spindle and chromatin as they are properly assembled in vivo. Chromatin near the centromere can be selectively labeled by inserting an array of DNA into the genome and expressing a fluorescent fusion protein that selectively binds to the insert. This can be achieved by inserting a repetitive DNA array containing monomers of the lactose (lacO) or tetracycline operator (tetO), into the genome and expressing a fluorescent fusion protein (lactose repressor (lacI) or tetracycline repressor (tetR) tagged with a fluorophore such as GFP that selectively binds to the insert [11]. Using wide-field epifluorescence microscopy, the diffraction limited spots of the operator bound to the repressor-GFP fusion protein can be observed over extended periods of time. Mean-squared displacement analysis can be applied to characterize the motion of the DNA and other spindle components in order to construct a polymer model.

Previous observations of the lacO spots in the mitotic spindle revealed clear asymmetries in the positioning of the sister chromatids, particularly in chromatin near the centromere. We 
propose that a more realistic model of pericentric chromatin includes two springs composed of chromatin near the centromere, one on either side of the paired chromatid arms that extend away from the core of the spindle. A drag force may act upon the arms, resulting in asymmetries that would not be predicted with a simple linear spring model.

\section{Materials and Methods}

\subsection{Polymer recoil in mitosis}

Cells were grown and imaged according to procedures laid out in Harrison, et. al, 2009 [12].

\subsection{Computational Modeling of the Mitotic Spindle}

An initial model computational model of the mitotic spindle was created by Ms. Leandra Vicci (Department of Computer Science, UNC Chapel Hill) in the Simulink modeling environment (unpublished). This model integrated microtubule dynamics, microtubule protein motor forces, and included compensation for the incredibly viscous intracellular environment. The force-response of chromatin was modeled as a linear spring (Fig. 2A).

Since DNA is the major component of chromatin, it follows that the behavior of DNA should serve as the basis for a higher-order model of chromatin. The worm-like chain (WLC) equation is commonly used to describe the mode of motion of polymers like DNA [13].

$$
F=\left(k_{B} * \frac{T}{L_{p}}\right)\left\lceil\frac{1}{4}\left(1-\frac{x}{L_{c}}\right)^{-2}-\frac{1}{4}+\frac{x}{L_{c}}\right\rceil
$$

In WLC, the amount of force $(F)$ that a polymer exerts per length stretched increases as extension $(x)$ increases. The WLC equation contains two key parameters for characterizing the spring. The first is persistence length $\left(L_{p}\right)$. If a polymer is shorter than $L_{p}$, it can be treated as a rigid body. The second parameter is contour length $\left(L_{c}\right)$. This is the total length of the polymer. Boltzmann's constant, $k_{B}$, represents the amount of thermal energy in the system when multiplied by temperature, $T$.

Using an embedded equation block in Simulink, we replaced the linear spring used to describe chromatin with the WLC equation. Chromatin is an aggregate of DNA and associated proteins. To begin to more realistically emulate the behavior of chromatin, we incorporated nucleosome binding and unbinding. Data from Fluorescence Recovery after Photobleaching (FRAP) shows that nucleosomes are dynamic within the pericentric chromatin (Verdaasdonk and Bloom, unpublished). Nucleosomal unbinding was modeled by increasing $L_{c}$ of the chromatin by $65 \mathrm{~nm}$, the approximate length of DNA bound by a single nucleosome. Binding was modeled by decreasing $L_{c}$ by the same length. The program is as follows:

$$
\begin{aligned}
& \text { function }[\mathrm{F}, \mathrm{Lc} 1]=\text { WLCspring }(\mathrm{x}, \mathrm{Lc}, \mathrm{Lp}) \\
& \% \text { Inputs: } \mathrm{x}=\text { extension length } \\
& \% \text { Lc=contour length } \\
& \% \mathrm{Lp}=\text { persistence length } \\
& \% \text { Outputs: } \\
& \% \mathrm{~F}=\text { Force } \\
& \% \text { Lc1=contour length }
\end{aligned}
$$




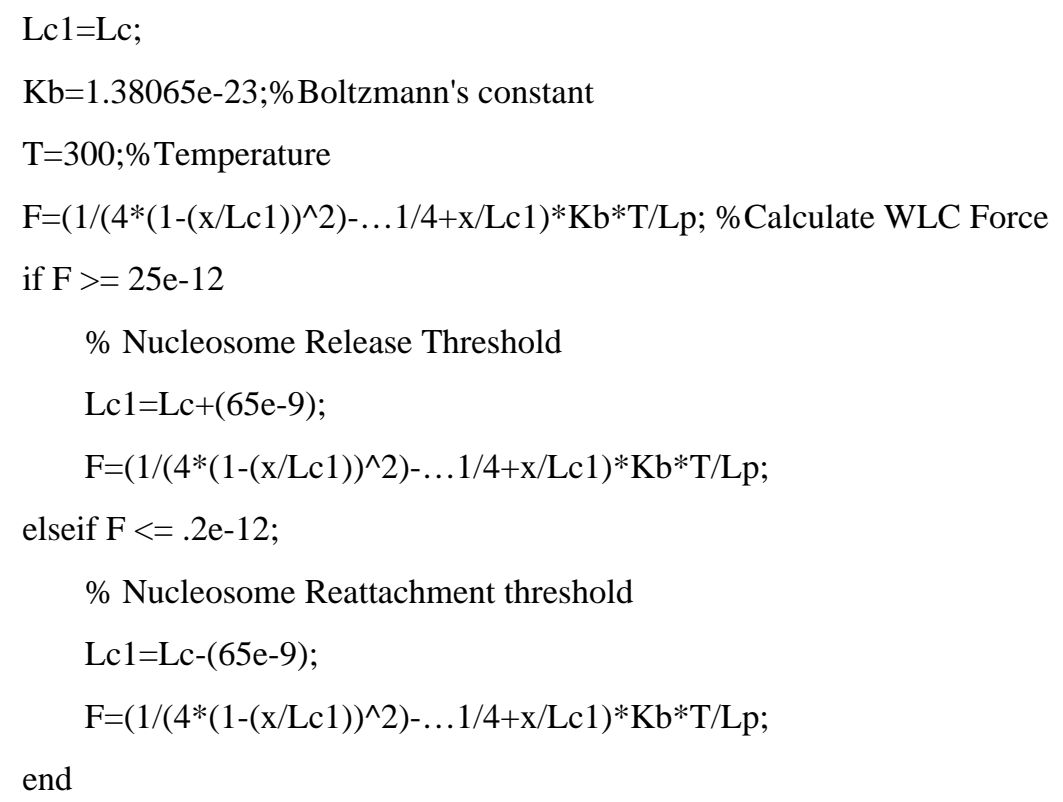

In order to explore the possibility that there are alternative forces acting to dampen the motion of the chromosomes during metaphase, we adapted an existing model of a dampedspring-mass system created by Leandra Vicci (UNC Department of Computer Science) to accept numerous parameters as adjustable inputs. This system accepts a perturbation and utilizes feedback to bring the object to rest. It utilizes mass, spring constant, and friction coefficient to demonstrate a damped system.

\subsection{Polymer modeling of Chromatin}

A strain of S. cerevisiae, KBY2531 [11], containing Spc29-GFP fusion protein to mark the spindle pole bodies, a 36x array of the lactose operator (lacO) inserted into the met14 locus near cen11, and a lacI-GFP fusion protein was used. During metaphase, four fluorescent spots are visible within this strain: two that demarcate the spindle pole bodies and two that demarcate the chromatin near the centromere of chromosome XI.

2.3.1 Cell Growth-KBY2531 cells were allowed to grow to logarithmic phase overnight at $24^{\circ} \mathrm{C}$ in $5 \mathrm{~mL}$ cultures in glass culture tubes in YPD, a complete, dextrose based media. Two milliliters of this culture was transferred to a $100 \mathrm{~mL}$ Erlenmeyer flask and diluted with $3 \mathrm{~mL}$ YPD. This culture was allowed to grow for 45 minutes at $24^{\circ} \mathrm{C}$. About $2.5 \mathrm{~mL}$ of this culture was pelleted, resuspended in $2.5 \mathrm{~mL}$ ddH20, and pelleted again. The pellet was resuspended in $5 \mathrm{~mL}$ SD-his media to promote transcription of lacI-GFP, transferred to a clean $150 \mathrm{~mL}$ Erlenmeyer flask, and allowed to grow for $60-90$ minutes at $24^{\circ} \mathrm{C}$. $3 \mathrm{AT}$, a drug used to induce expression of the His promoter, was then added to a final concentration of $20 \mathrm{nM}$, and the culture was shaken for another 30 minutes at $24^{\circ} \mathrm{C} .1 \mathrm{~mL}$ of this culture was transferred to a $1.4 \mathrm{~mL}$ eppendorf tube, pelleted, and resuspended in $1 \mathrm{~mL}$ ddH20. This was again pelleted and resuspended in $\sim 300 \mathrm{uL} \mathrm{ddH} 20$. Seven microliters of this solution was placed onto YC complete slab media.

2.3.2 Imaging-Single plane images were taken using a Nikon TE2000-U microscope with a 1.4 NA 100xs objective, Hamamatsu Orca ER CCD Camera, and $400 \mathrm{~ms}$ exposure time at five second intervals.

2.3.3 Data analysis-Time-lapse photo series were loaded into a novel graphical user interface (GUI) based analysis tool designed in MatLab. If necessary, a smoothing algorithm 
was applied to help differentiate the spots from background noise. The centroids of the spots were calculated by selecting a spot radius and rough center by hand and then fitting the selected region with a 2-dimensional Gaussian distribution to obtain sub-resolution estimates of spot location [14]. The spots within each image in the photo series were selected by hand and the coordinates were stored for later use. Spots that were out of focus or otherwise unclear were not selected.

The coordinates of the four fluorescent spots were then translated into a moving reference frame to compensate for possible cellular motion or unbalanced cellular forces that may disturb the position of the mitotic spindle. This was done by defining the $\mathrm{x}$-axis as the line running through the centroids of both spindle pole bodies. The origin was defined as the midpoint between the spindle pole bodies.

Mean square displacessment analysis was then conducted by implementing the following formula:

$$
<(x(t)-x(t+\tau))^{2}>
$$

where $\mathrm{x}(\mathrm{t})$ is the $\mathrm{x}$-coordinate of the spot at time $\mathrm{t}$ and $\mathrm{x}(\mathrm{t}+\tau)$ is that same spot's position at $\tau$ seconds later. This analysis was conducted for the spindle pole bodies, the DNA spots relative to the spindle pole bodies, and the DNA spots relative to the origin.

The non-Gaussian parameter for both spindle pole bodies and DNA spots was calculated with the following formula [15]:

$$
\frac{<(x(t)-x(t+\tau))^{4}>}{3<(x(t)-x(t+\tau))^{2}>^{2}}-1
$$

This analysis was implemented to determine whether the movement of a given object can be explained by thermal diffusion or if there are more complex processes acting on it. In both the MSD analysis and non-Gaussian parameter analysis, $\tau$ varied from the smallest time step possible for a given photo series (time lag between frames) to about one third of the total photo series time. Note that both of these analyses are prone to noise at higher values of $\tau$ because fewer data points can be included in the averages.

\section{Results}

\subsection{Polymer recoil in mitosis}

The ability to place arrays of Lac-operator at different chromosomal loci provides the opportunity to examine the behavior of these loci as they segregate in live cells. The pericentric chromatin DNA tracks with depolymerizing microtubule plus-ends in anaphase. In contrast, distal loci (hundreds of kilobase pairs away from the centromere) exhibit very different kinetics during anaphase [12]. DNA spots at the ends of replicated chromosomes (telomeres) do not separate until they come to lie proximal to the mitotic spindle (see Fig. 3 ). Once along the spindle axis, the spots become visibly separated ( 90sec, Fig. 3) and one of the sister telomeres moves to the pole more rapidly than the rate of pole separation (about $\sim 5 \times$ the rate of pole separation). At this stage there is no microtubule depolymerization at the kinetochore. Thus this rapid rate of movement reflects elastic recoil of the telomere to the spindle pole. 
3.1.1 Polymer recoil after chromosome breakage in vivo-To investigate the elastic nature of chromatin under a different cellular condition, we made use of conditionally dicentric chromosomes [7]. Two centromeres (one conditionally functional) were introduced onto the same chromosome (chromosome III). In mitosis, DNA between the two centromeres is under tension when the centromeres on the same sister chromatid are activated and allowed to attach to opposite spindle poles. In this situation tension on the DNA is not dependent upon sister chromatid cohesion (as above), rather tension is exerted along one contiguous DNA helix. Previous studies have found that the spindle can generate enough force to stretch the DNA between the two centromeres to the B-form length $(0.34 \mathrm{~nm} / \mathrm{bp})[16]$. In rare cases, these stretched dicentric chromosomes can break (Fig. 4). After a breakage event, the chromatin recoils to its unbroken end, exhibiting different rates of movement during different phases of recoil. From the representative breakage/recoil event in Figure 4, the initial fast phase of recoil occurs at approximately $1.53 \mu \mathrm{m} / \mathrm{min}$ followed by a slower phase of $0.48 \mu \mathrm{m} / \mathrm{min}$. The biphasic nature of chromatin recoil observed in vivo is also observed in naked DNA recoil in vitro [7]. The observation of chromatin recoil after dicentric chromosome breakage suggests that chromatin has inherent elastic properties, due in part to the entropic elasticity of DNA.

\subsubsection{Perturbation of chromatin compaction leads to visible chromatin} stretching-Chromatin recoil may also reflect chromatin compaction following force extension. To investigate the role of chromatin compaction in stretching and elastic recoil, we studied telomere separation in cells deficient in several proteins involved in higher order chromatin structure, the histone deacetylase, Sir2 and topoisomerase I. Both of these proteins have been implicated in either establishment and/or maintenance of chromosome condensation [16,17]. In both strains lacking these proteins ( $\operatorname{sir} 2 \Delta$ and top $1 \Delta$ ), the lacO array on a single sister chromatin exhibited substantial stretching in anaphase (Fig. 5). Stretching occurred just after the initial separation of sister telomere-proximal lacO arrays. $\operatorname{sir} 2 \Delta$ cells showed this phenotype in $\sim 14 \%$ (1/6) cells where top $1 \Delta$ showed stretching in $\sim 40 \%$ (3/7) of cells (Fig. 5). Single sister lacO spot stretching was not observed in telophase nor G1 in these strains. Additionally, single sister telomere lacO spot stretching was not observed in wild type cells (0/11). This phenotype indicates that SIR2 and TOP1 play a role in chromatin condensation at telomeres. These observations also suggest that during anaphase the ends of the chromatid polymer (telomeres) are under tension, and that tension is of sufficient magnitude to decompact chromatin in these mutant cellular backgrounds.

\subsection{Computational Modeling of the Mitotic Spindle}

We constructed a Simulink block that behaves like WLC (see Materials and Methods). When nucleosomal disruption events were taken into consideration, the force curve took on a sawtooth conformation. This was similar in shape to nucleosome disruption curves observed in vitro [9], confirming the behavior of the model (Fig. 6).

When the WLC spring block was inserted into the larger model of the mitotic spindle, the overall dynamics of the model were unaffected. In both situations, overall spindle length grew steadily. The average total force exerted by the chromatin "spring" was greatly reduced in the WLC model. However, this can be rectified by adjusting parameters within the WLC equation.

The damped-spring-mass system used to examine the role of feedback within a process demonstrated oscillatory behavior. Parameters could be adjusted to damp oscillations or create oscillations that grow out of control. This model was not built with the intent of connecting it to the model of the mitotic spindle. 


\subsection{Polymer Modeling of Chromatin}

A linear regime was previously observed in MSD traces for both spindle pole bodies and lacO spots in KBY2531 (Stephens and Bloom, unpublished). Five photo series were taken at 5 second time steps to most accurately capture the linear portion of these analysis curves. The linear portion of these curves reflects consistent mode of motion over time and allows one to deduce the physical behavior over this time period. Note that these curves appear linear on log-log plots, so the geometric average was taken to find a consensus line for both the spindle pole bodies and the DNA spots. This revealed a slope of 0.5 (decades of microns)/(decades of seconds) for the averaged spindle pole bodies and a slope of 0.25 (decades of microns)/(decades of seconds) for the averaged DNA spots when the square root of MSD was plotted against time step (Fig. 7).

Non-Gaussian parameters were calculated for all possible values of $\tau$ and plotted against $\tau$ for the spindle pole bodies and the DNA spots for the five photo series taken at 5 second time steps. Not all exhibited similar behavior, so average curves were not calculated. However, for low values of $\tau$, neither the spindle pole bodies nor the DNA spots had steady values of 0 , indicating that they were not undergoing random diffusion [18] (Fig. 8). Note that some DNA plots exhibit radically different behavior, suggesting that there may be several distinct modes of motion for the DNA spots.

\section{Discussion}

\subsection{Polymer recoil in mitosis}

In previous works, the observation of bi-orientation of a chromosome in mitosis was used as an indicator of tension [19]. In this study, our observation of chromatin recoil indicates that chromatin is indeed under tension during cell division and that the chromosomes recoil elastically when perturbed. Thus, observations of chromatin recoil demonstrate that it is indeed under tension and that chromatin can act as a force-providing spring within the construct of the mitotic spindle. Furthermore, the increased incidence of lacO stretching in cells where chromatin structure is altered indicates that chromatin structure is essential to defining the characteristics of chromatin as a spring.

\subsection{Computational Modeling of the Mitotic Spindle}

Computational modeling of any biological process is highly dependent on proper parameterization to attain a model that is both biologically relevant and predictive of future experiments. However, models must be simple enough to present the process in an easilyunderstood framework. Thus there are two important paradoxes: models must be simple enough to understand but must be complex enough to be realistic, and models must be based on experimental evidence but also predict the outcome of future experiments.

Creating a computational model of the mitotic spindle is truly a monumental task. Even though many parameters are defined (such as the force required to stall a kinesin motor protein), many of these experiments were conducted in vitro. It is impossible to accurately replicate the intracellular environment in vitro. At best, these quantities can be used only as rough estimates of their counterparts within a cell. With a high level of uncertainty in parameters, a model can only be used to make general behavioral observations.

Although inserting the WLC behavior for chromatin into the model of the mitotic spindle did not significantly alter model behavior, the WLC model was able to recreate a previously observed force-extension curve for nucleosome disruption (Fig. 6C and D). This suggests that the model could certainly be more accurate than a simple linear spring. However, the lack of response by the model of the mitotic spindle suggests that the exact method of how 
chromatin responds to and/or generates force is inconsequential. This is obviously untrue, as chromatin is a highly ordered and regulated structure. Any perturbation of chromatin (i.e. the application of force) could potentially damage it. Thus, this result suggests that the model of the mitotic spindle is underdeveloped and that it may not yet be sensitive enough to utilize more complicated models of chromatin.

Although this model is not yet fully developed, it provides a valuable framework within which to examine the relationships of known components of the mitotic spindle. Although it may not be able to predict experimental results, points of weakness within the model point to experiments that can be done to better characterize the mitotic spindle.

The damped-spring-mass system provides an interesting framework within which to consider the movement of chromosomes. Although the parameters used to explore this system were not necessarily biologically relevant, it demonstrates a system in which a perturbation can be applied to an object, causing that object to oscillate. The dynamics of those oscillations are controlled by the object's intrinsic properties, the properties of the surrounding environment, and feedback.

In the context of the mitotic spindle, a chromosome would represent the object while microtubules provide the perturbation [12]. Unlike a linear spring model or even the WLC model, the chromosome would not necessarily respond symmetrically to a perturbation if there was an outside damping force applied to the chromosome such as viscous drag. Thus, this model can be used to explain asymmetry observed in the mitotic spindle at a basic level and should be expanded upon and incorporated into future models of chromatin.

Feedback is found in most biological processes, so it follows that it could be an integral part of regulation of the mitotic spindle and of the mitotic spindle cell cycle checkpoint. Although common feedback loops in biology are chemically based, it is also possible for feedback to be physically or mechanically based. One important element of a feedback loop is a reference input-an ideal value toward which the feedback loop attempts to guide the process that is being regulated. In many biological processes this value is usually an ideal concentration of a substrate or biomolecule. In the case of the mitotic spindle, however, this input could be tension. Proper tension has already been implicated as a pre-requisite for anaphase onset, and Ndc80, a protein in the kinetochore, has been shown to change conformation in the presence of tension [20,21]. A similar tension-sensing mechanism could potentially trigger chromatin remodeling complexes, resulting in a tension-based feedback loop. This feedback loop could partially explain chromatin spot movement and spindle length fluctuation within the mitotic spindle. It can reasonably be predicted that any perturbation to this feedback loop, such as the removal of chromatin structural proteins, would cause exaggerated fluctuations, much like the perturbation of a parameter in our damped-spring-mass feedback model can cause exaggerated oscillations.

\subsection{Polymer Modeling of Chromatin}

The linear fits to the spindle pole spots and DNA spots are indicative of the type of motion that these spots undergo (Fig. 7). These slopes indicate that the spindle pole bodies are undergoing diffusive motion while the motion of the DNA spots is sub-diffusive. However, the non-Gaussian parameter plots suggest that the spindle pole bodies are in fact not undergoing completely random diffusion (Fig. 8). This suggests that further analysis must be done to fully describe that mode of motion that the spindle pole bodies undergo. It has been shown that processes that appear to be diffusive at first glance may not actually be diffusive, thus these results are not particularly troublesome [10]. 
The sub-diffusive nature of the DNA spots relative to their respective spindle pole bodies is indicative of some sort of restoring force on the DNA. Under the assumption that these spots are discrete points of no weight, this restoring force could be attributed to tension in the across the chromosome. This is a fair hypothesis because it is known that there are mechanical linkages connecting the sister chromatids to their respective spindle pole bodies (microtubules and kinetochores) as well as to one another (cohesin). It has commonly been thought that the cohesin linking sister chromatids dissociates at the onset of anaphase.

Recent evidence, however, suggests that there are persistent mechanical linkages between the sisters during anaphase [12]. If such linkages exist, we can reasonably predict that the DNA spots will present with a smaller slope when compared to the spindle pole bodies in anaphase.

Sub-diffusive behavior has also been observed in molecules that experience molecular crowding [22]. Due to the incredibly dense nature of the intracellular environment, it is very reasonable to infer that molecular crowding may be the cause of the sub-diffusive nature of these lacO spots. However, the close proximity of these spots to the centromere cannot be ignored. It is very possible that tensile forces on chromatin near the centromere could cause spot motion that is greater than diffusion (directed motion). If the array of lacO formed a loop that was somehow exempted from the tensile forces running through the chromatin near the centromere, molecular crowding could explain the sub-diffusive nature of these spots regardless of the behavior of the chromatin under tension.

The non-Gaussian parameters also suggest that not all DNA spots undergo the same behavior. This may indicate that not all DNA spots are being acted upon in the same manner by the cell. Thus, the non-Gaussian parameter plots may be able to be used to group the DNA spots by mode of behavior. Biologically, this can be explained by the dynamic nature of the microtubules. A kinetochore microtubule can be growing (rescue), shrinking (catastrophe), or stable (pause). Each chromosome is affected by two microtubules, resulting in six distinct permutations of microtubule behavior. Further work needs to be done determine the effect of microtubules on the DNA spots. Alternatively, cells could be imaged in the presence of a microtubule stabilizing drugs to negate the effects of microtubule dynamics.

\subsection{The Role of Chromatin within the Mitotic Spindle}

At this point in time, it is impossible to explain chromatin's exact response to force. The structure of chromatin is largely unknown. This research, however, is consistent with the hypothesis that chromatin does not necessarily respond as a linear spring when force is applied. In simple models of the mitotic spindle, a linear spring model of chromatin may be sufficient to yield usable results. However, as our understanding of the mitotic spindle grows, this assumption will become invalid and the exact behavior of chromatin will become increasingly more important to future models.

Characterizing chromatin as a polymer is a simplistic way to create a model of chromatin without having to consider every protein complex associated with it individually.

Eventually, this may lead to a mathematical formula that can be used to describe chromatin, much like the WLC equation has been used to describe DNA. Systematic mutations could then be made to proteins that are implicated in chromatin structure. The polymer model would provide a framework within which to characterize each of these complexes.

The implication of tension in the pericentric chromatin during metaphase as a trigger for cell cycle progression makes metaphase the optimal phase in which to build preliminary models of pericentric chromatin. However, the structure of chromatin is important throughout the 
cell cycle. Understanding of chromatin structure in metaphase will lead to insights about chromatin structure throughout all of mitosis and throughout the entire cell cycle.

\section{Acknowledgments}

We would like to thank Dr. Michael Rubinstein (Dept of Chemistry, UNC-CH) and Ms. Leandra Vicci (Dept of Computer Sci, UNC-CH) for their helpful discussion. This project was supported in part by the James Henley Thompson and Evelyn Barnett Thompson Undergraduate Research Fund, administered by the Honors Office at UNC-Chapel Hill to M.E.L. and NIH GMR01 32238-24 to K.B.

\section{References}

1. Mitchison TJ, Salmon ED. Mitosis: a history of division. Nat Cell Biol 2001;3:E17-E21. [PubMed: 11146645]

2. Hoyt MA, He L, Loo KK, Saunders WS. Two Saccharomyces cerevisiae kinesin-related gene products required for mitotic spindle assembly. J Cell Biol 1992;118:109-120. [PubMed: 1618897]

3. Joglekar AP, Bouck DC, Molk JN, Bloom KS, Salmon ED. Molecular architecture of a kinetochoremicrotubule attachment site. Nat Cell Biol 2006;8:581-585. [PubMed: 16715078]

4. Peterson JB, Ris H. Electron-microscopic study of the spindle and chromosome movement in the yeast Saccharomyces cerevisiae. J Cell Sci 1976;22:219-242. [PubMed: 794073]

5. Clarke L, Carbon J. Isolation of a yeast centromere and construction of functional small circular chromosomes. Nature 1980;287:504-509. [PubMed: 6999364]

6. Bouck DC, Bloom K. Pericentric chromatin is an elastic component of the mitotic spindle. Curr Biol 2007;17:741-748. [PubMed: 17412588]

7. Fisher JK, Ballenger M, O'Brien ET, Haase J, Superfine R, Bloom K. DNA relaxation dynamics as a probe for the intracellular environment. Proc Natl Acad Sci U S A 2009;106:9250-9255. [PubMed: 19478070]

8. Gardner MK, Pearson CG, Sprague BL, Zarzar TR, Bloom K, Salmon ED, Odde DJ. Tensiondependent regulation of microtubule dynamics at kinetochores can explain metaphase congression in yeast. Mol Biol Cell 2005;16:3764-3775. [PubMed: 15930123]

9. Brower-Toland BD, Smith CL, Yeh RC, Lis JT, Peterson CL, Wang MD. Mechanical disruption of individual nucleosomes reveals a reversible multistage release of DNA. Proc Natl Acad Sci U S A 2002;99:1960-1965. [PubMed: 11854495]

10. Wang B, Anthony SM, Bae SC, Granick S. Anomalous yet Brownian. Proc Natl Acad Sci U S A 2009;106:15160-15164. [PubMed: 19666495]

11. Pearson CG, Maddox PS, Salmon ED, Bloom K. Budding yeast chromosome structure and dynamics during mitosis. J Cell Biol 2001;152:1255-1266. [PubMed: 11257125]

12. Harrison BD, Hoang ML, Bloom K. Persistent Mechanical Linkage Between Sister Chromatids Throughout Anaphase. Chromosoma 2009;118:633-645. [PubMed: 19603176]

13. Bustamante C, Marko JF, Siggia ED, Smith S. Entropic elasticity of lambda-phage DNA. Science 1994;265:1599-1600. [PubMed: 8079175]

14. Thompson RE, Larson DR, Webb WW. Precise nanometer localization analysis for individual fluorescent probes. Biophys J 2002;82:2775-2783. [PubMed: 11964263]

15. Odagaki T, Hiwatari Y. Gaussian-to-non-Gaussian transition in supercooled fluids. Phys Rev A 1991;43:1103-1106. [PubMed: 9905128]

16. Thrower DA, Bloom K. Dicentric chromosome stretching during anaphase reveals roles of Sir2/Ku in chromatin compaction in budding yeast. Mol Biol Cell 2001;12:2800-2812. [PubMed: 11553718]

17. Goto T, Wang JC. Cloning of yeast TOP1, the gene encoding DNA topoisomerase I, and construction of mutants defective in both DNA topoisomerase I and DNA topoisomerase II. Proc Natl Acad Sci U S A 1985;82:7178-7182. [PubMed: 2997777]

18. Rahman A, Singwi KS, Sjolander A. Theory of slow neutron scattering by liquids. Physical Review 1962;162:986-996. 
19. Nicklas RB. The forces that move chromosomes in mitosis. Annu Rev Biophys Biophys Chem 1988;17:431-449. [PubMed: 3293594]

20. Dewar H, Tanaka K, Nasmyth K, Tanaka TU. Tension between two kinetochores suffices for their bi-orientation on the mitotic spindle. Nature 2004;428:93-97. [PubMed: 14961024]

21. Joglekar AP, Bloom K, Salmon ED. In vivo protein architecture of the eukaryotic kinetochore with nanometer scale accuracy. Curr Biol 2009;19:694-699. [PubMed: 19345105]

22. Weiss M, Elsner M, Kartberg F, Nilsson T. Anomalous subdiffusion is a measure for cytoplasmic crowding in living cells. Biophys J 2004;87:3518-3524. [PubMed: 15339818] 


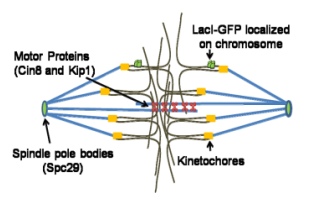

Figure 1. Schematic of the mitotic spindle in Saccharomyces cerevisiae

Microtubules extend inward from the spindle pole bodies. They attach to replicated sister chromatids via kinetochores or are cross-linked by microtubule motor proteins. Multiple arrays of the lactose operator DNA binding site, coupled with a lactose repressor-GFP fusion protein, can be used to visualize chromatin in a living cell. In the cell, sixteen pairs of sister chromatids are organized in a circular fashion around the overlapping interpolar microtubules in the center. Legend: spindle pole bodies (green circles), microtubules (blue lines), chromatin (gray lines), kinetochore (yellow square), kinesins (red ovals), and lacIGFP (small green circles). 


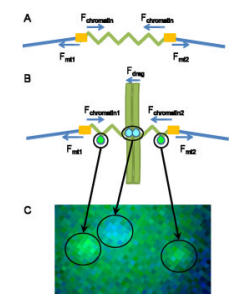

Figure 2.

(A) Classic model of microtubule force and chromosome response. A chromatin spring provides the only resistance to microtubule force. (B) Updated model of microtubule force and chromosome response. Chromatid arms provide a viscous drag force, allowing asymmetrical behavior in pericentric chromatin. (C) Image of asymmetry in pericentric chromatin in S. cerevisiae. Green dots represent chromatin near kinetochores while the blue dot represents chromatin at the intersection of the pericentric chromatin and the chromatid arms. 


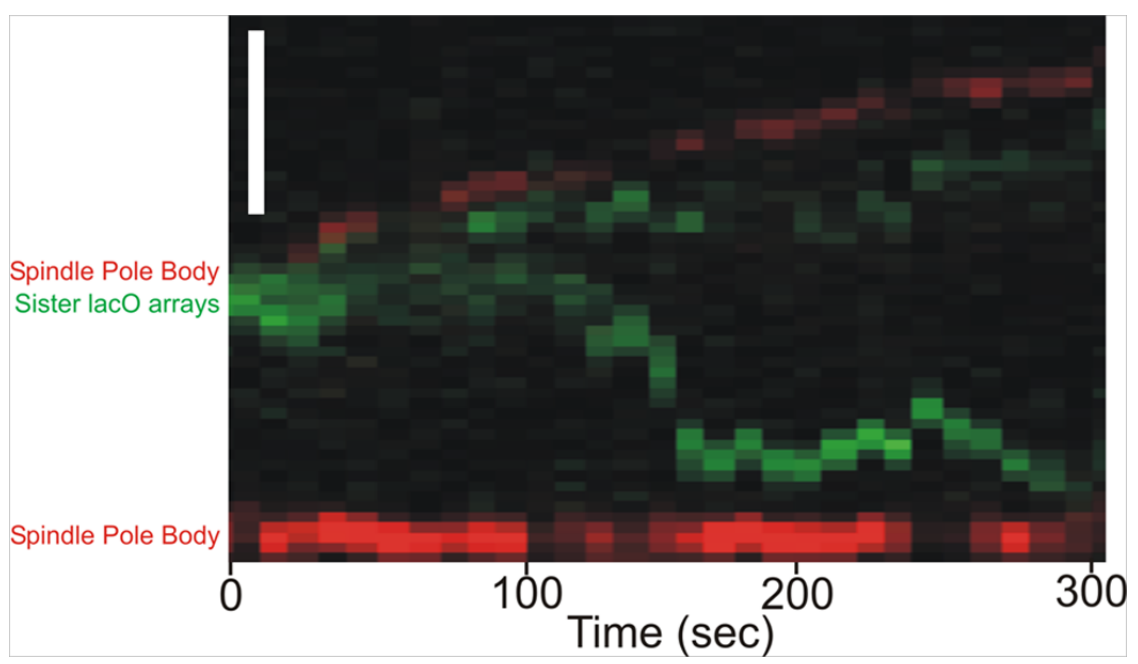

Figure 3. Asymmetric Recoil Dynamics of Sister Telomeres

A representative kymograph of a time lapse image series of telomere-proximal lacO arrays (green) and spindle pole bodies (red). One spindle pole body was arbitrarily fixed in space to provide a frame of reference (MATLAB software developed in house). The slope of the top red line represents spindle elongation. The sister lacO arrays at the telomeres (green line) separate at about $90 \mathrm{sec}$. One sister chromatid recoils to the spindle pole as evidenced by the rapid rate of movement relative to spindle elongation (in red). In all time lapse series taken $(11 / 11)$, sister telomeres are asymmetrically positioned on the mitotic spindle and sister separation initiated between the spindle pole bodies. Upon sister separation, one of the sisters exhibits rapid recoil to the distal spindle pole. Kymograph based on images taken at $10 \mathrm{sec}$ intervals. Scale bar represents $2 \mu \mathrm{m}$. 


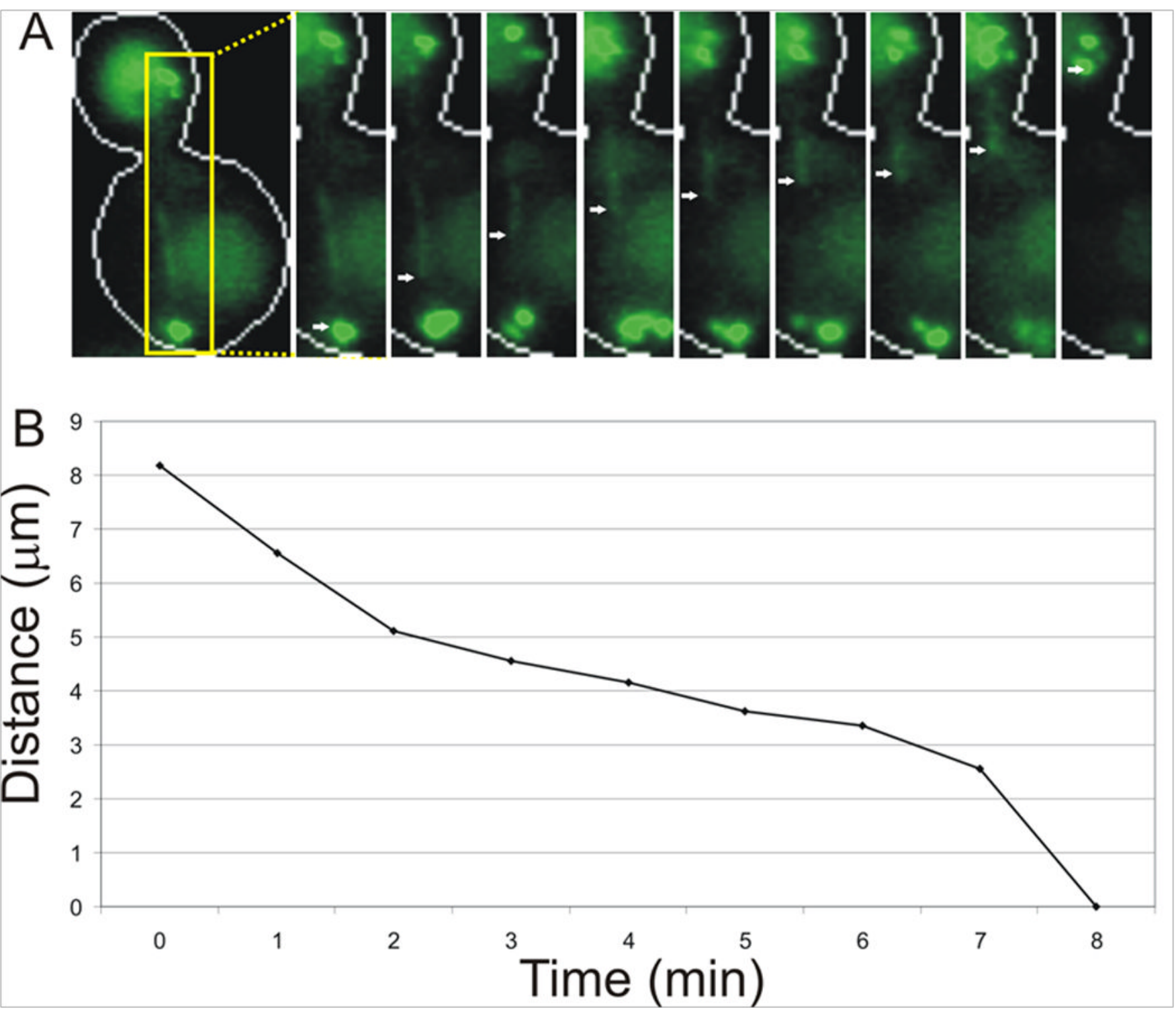

Figure 4. Recoil dynamics of a broken chromosome

DNA between two centromeres on a single chromosome (dicentric chromosome) can extend to its B-form length when the two centromeres on the same sister chromatid are attached to opposite spindle pole bodies. The DNA is visualized via multiple arrays of the lacO DNA binding site inserted between the two centromeres and bound by lac repressor-GFP. The yellow box in the first panel of (A) is blown up for panels 2-10. Panel 2 shows the chromatin polymer just prior to breakage. Arrows in panels 3-10 mark the end of the recoiling polymer. The length of the recoiling polymer in each panel is shown in (B). Images were taken at $1 \mathrm{~min}$ intervals. The rapid phase of recoil is approximately $2 \mathrm{microns} / \mathrm{minute}$. 

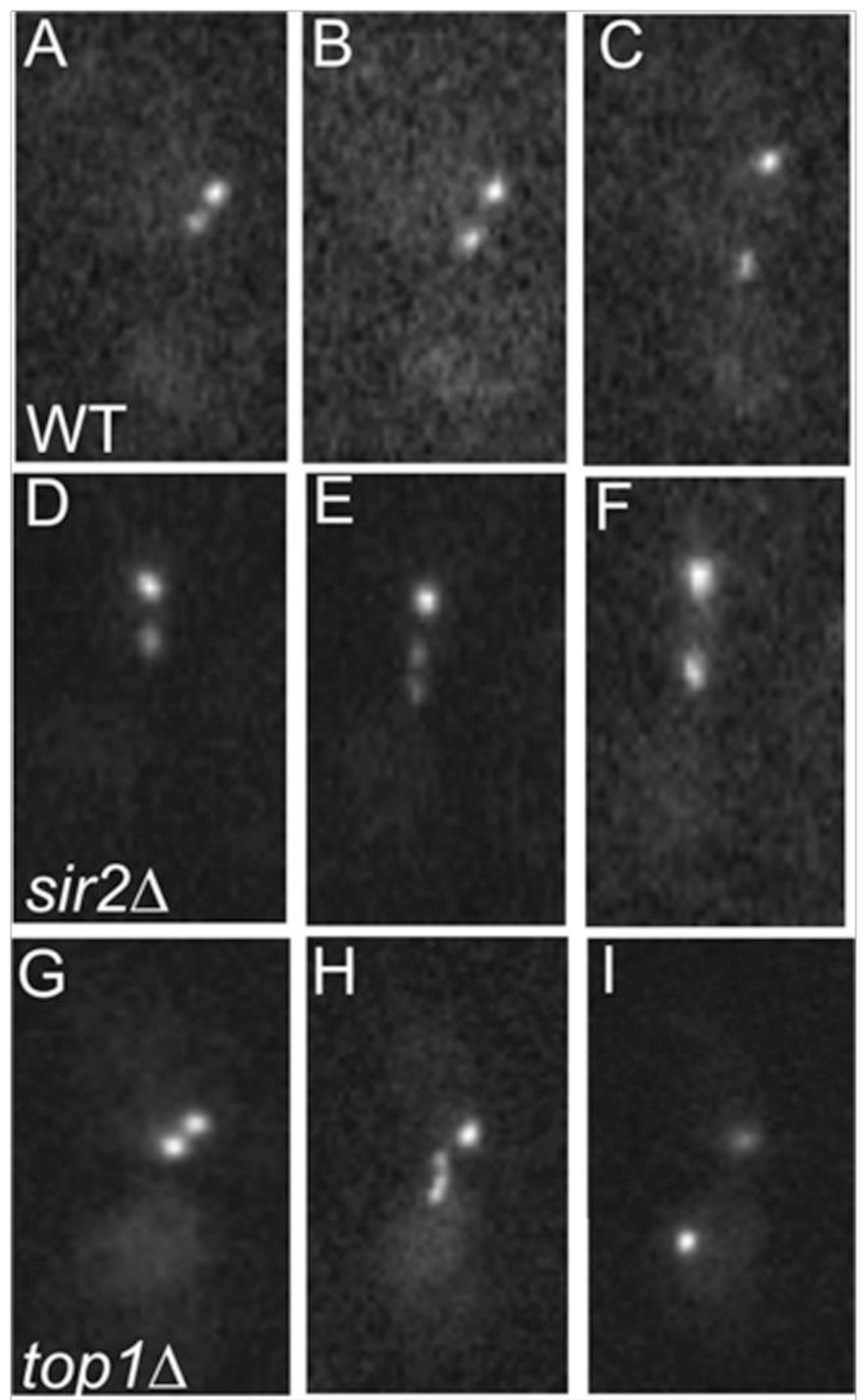

Figure 5. Telomere Stretching

Sets of three sequential images taken at $10 \mathrm{sec}$ intervals depicting sister telomere-proximal lacO arrays immediately following lacO array separation. In a wild type background, sister telomeres separate without either sister lacO array displaying stretching (A-C). In a sir2 4 mutant (D-F), one of the lacO array stretches (bottom), becoming two spots $10 \mathrm{sec}$ after the sister lacO arrays appear separated $(\mathrm{E})$. In a top $1 \Delta$ mutant $(\mathrm{G}-\mathrm{I})$, one of the sister lacO arrays (bottom) stretches to form a bar $(\mathrm{H})$. 


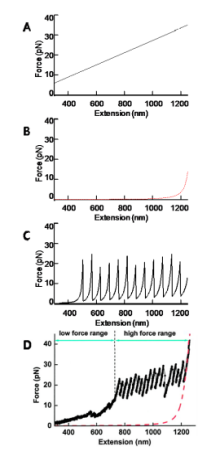

Figure 6.

(A) Plot of Force v. Extension curve for a simple linear spring. (B) Plot of Force v. Extension for a Worm-Like Chain meant to simulate DNA. (C) Plot of Force v. Extension for a Worm-Like Chain with adjustable contour length. Each dip simulates the release of one nucleosome from DNA. (D) Experimental data from Brower-Toland B D et al. ${ }^{9}$ showing the Force v. Extension curve for DNA and nucleosomes. 


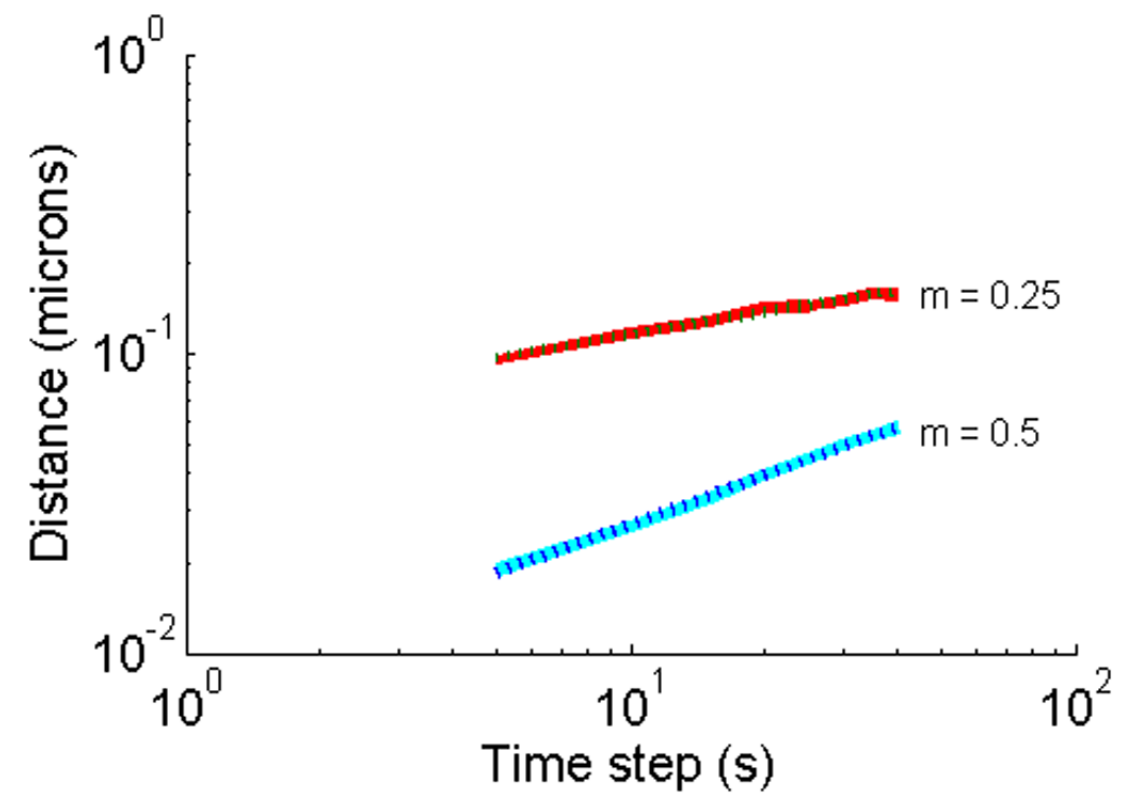

Figure 7.

Plot of root mean square displacement v. time step $(\tau)$ for the linear consensus region as determined from Stephens and Bloom, unpublished. Linear fits overlay the average values from five different data sets. The red line indicates the geometric average of lacO spot movement (at 1.1 kilobases from cen11) while the blue line indicates the geometric average of spindle pole body movement. Overlaid dotted lines represent linear fits, with slope (m) given in decades of microns per decades of seconds. 


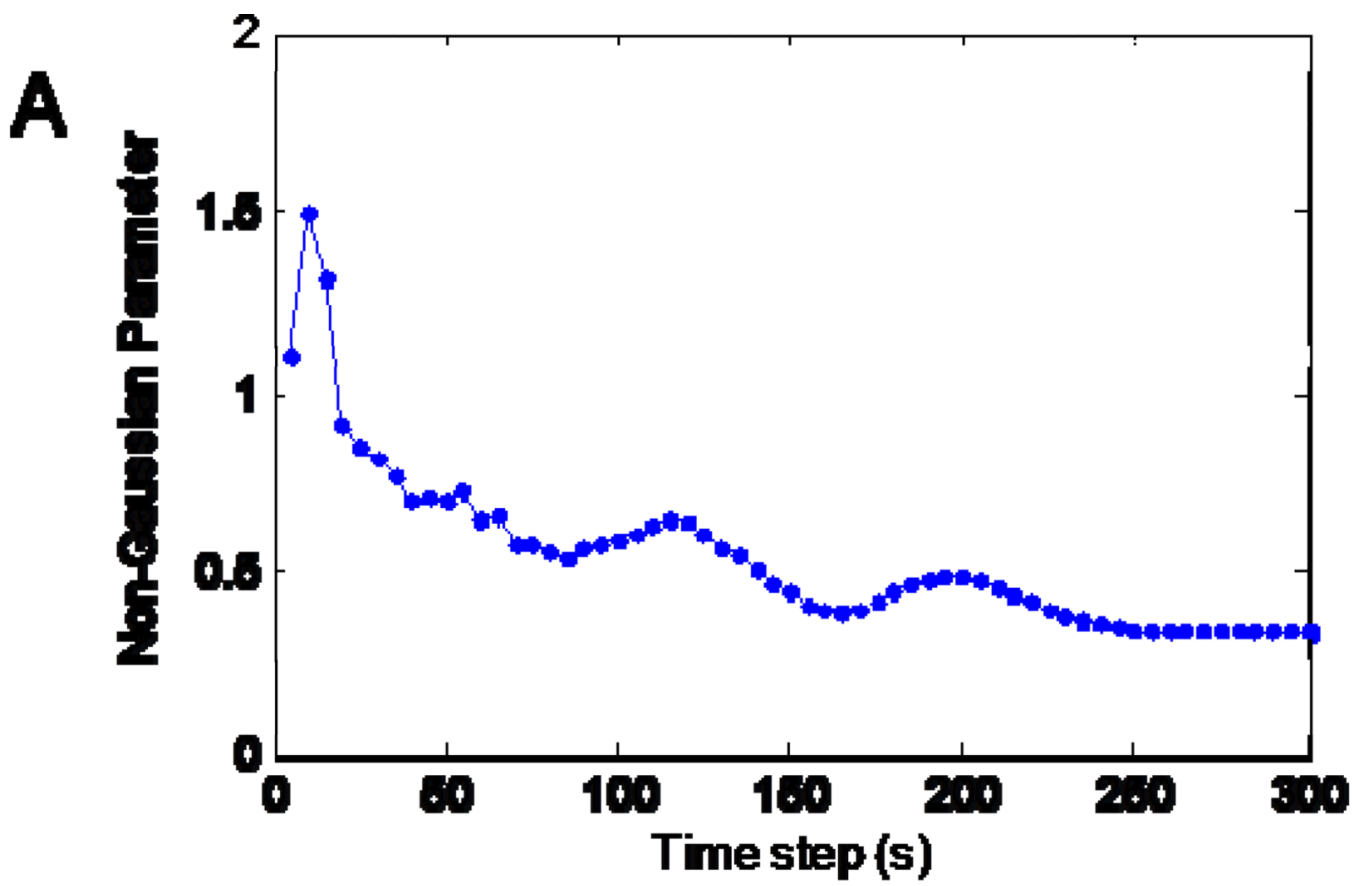

B

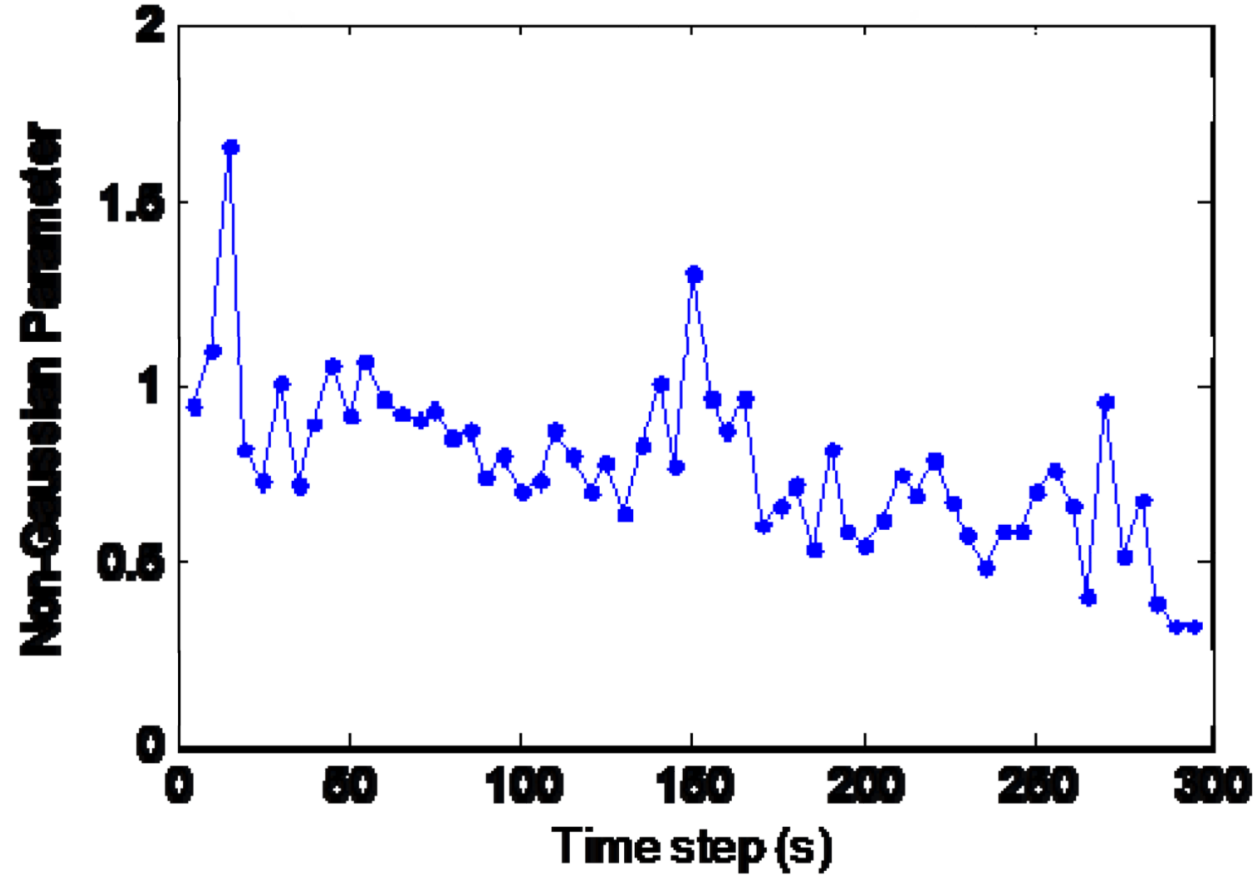

Figure 8.

Plots of non-Gaussian parameter v. time step. Blue lines indicate the non-Gaussian parameter of the indicated fluorescent spot while red lines indicate the expected nonGaussian parameter for a diffusive process ${ }^{18}$.

(A) Plot of non-Gaussian parameter v. $\tau$ for spindle pole bodies. (B) Plot of non-Gaussian parameter $\mathrm{v}$. $\tau$ for DNA spots. 